\title{
Dynamic Economic Dispatch for Wind Power System Considering System Security and Spinning Reserve
}

\author{
Wangchao Dong, Jing Zhang, Jiejie Huang, Shenghu Li \\ School of Electric Engineering and Automation, Hefei University of Technology, Hefei, China \\ Email: dwchhf@live.com
}

Received December 2014

\begin{abstract}
In this paper, dynamic economic dispatch model is proposed for power systems with bulk wind power integration. The wind turbine generators are assumed to partially undertake the spinning reserve for the thermal generator. A double-layer optimization model is proposed. The outer layer use the differential evolution to search for the power output of thermal generators, and the inner layer use the primal-dual interior point method to solve the OPF of the established output state. Finally, the impact of spinning reserve with wind power on power system operating is validated.
\end{abstract}

\section{Keywords}

Spinning Reserve, Wind Power, Dynamic Economic Dispatch (DED), Differential Evolution Method, Interior Point Method

\section{Introduction}

The wind power is a kind of clean and sustainable energy source. However, considering the stochastic and intermittent properties, bulk integration of wind farms to the power systems may yield various problems to security and stability. To guarantee the reliability of power supply, reserve capacity as much as installed capacity of the wind power is necessary, which limited the development of wind power. At the mean time, there is a new challenge brought by randomness of wind power. It is difficult for existing wind speed forecasting technology to meet the reliability and accuracy requirement for traditional power generation plan.

To reduce the effects of wind power instability, many researches were proposed in the field of prediction accuracy of wind speed and spinning reserve of wind power. Reference [1]-[3] proposed advanced prediction method, which the root mean square error of wind power prediction is between $10 \%$ and $20 \%$. Reference [4] discussed the traditional calculation of reserve capacity which is easy to application. But it is not optimal considering economy and reliability. Reference [5] apply the optimization algorithm to calculate the optimal spinning reserve required and balance the reliability and economy of generators' outage, prediction deviation of load and wind power. Reference [6] [7] considered the spinning reserve cost and set the objective function as minimum total cost of generation and operation. Reference [8] [9] calculated the reserve capacity by using the reliability 
requirement of probability distributions of load and wind power prediction error. But it is not safe and economic for thermal power unit to supply spinning reserve considering the energy cost, efficiency, and the ramp speed.

The article applied the spinning reserve model for wind farm to reducing the capacity demand of system security. Based on spinning reserve model, the wind power system optimal scheduling considering wind turbines was established. The calculating result was compared with that reserve model ignored. Interior point nested differential evolution method was applied to solve the dynamic economic dispatch considering security constraints.

\section{Wind Farm Spinning Reserve Model}

\subsection{Wind Farm Output Optimal Control Strategy}

A new optimal method to achieve complementary spare between multiple wind farms is presented which can meet the requirement of high dimensions and multitasking scheduling. The method is expressed as follows:

$$
\begin{aligned}
& \max P^{\text {out }}=\sum_{j=1}^{D} \sum_{t=1}^{T} P_{j, t}^{\text {out }} \\
& \sum_{t=1}^{T} P_{j, t}^{\text {out }}<\sum_{t=1}^{T} P_{j, t}^{\text {set }} \\
& 0<P_{j, t}^{\text {out }}<P_{j, t}^{\text {pre }}
\end{aligned}
$$

where $D$ is number of wind farms; $P_{j, t}^{\text {out }}$ is real-time sampling output of wind farm $j$ at hour $t ; P_{j, t}^{\text {pre }}$ and $P_{j, t}^{\text {pre }}$ represent the given and predictive value of wind farm $j$ at hour $t$.

\subsection{Reserve Capacity Calculation}

Normal distribution and Laplace distribution are applied to establish the distribution $P_{d f}\left(e_{w p}\right)$ of wind power predictive error $e_{w p, t}$.

$$
e_{w p, t} \sim f_{T}\left(e_{w p}\right)=a_{1} f\left(e_{w p}\right)+a_{2} g\left(e_{w p}\right)
$$

where, $f\left(e_{w p}\right)$ and $g\left(e_{w p}\right)$ represent the density functions of Normal distribution and Laplace distribution; $a_{1}$ and $a_{2}$ meet the following requirement.

$$
\left\{\begin{array}{l}
3 a_{1}+6 a_{2}=k_{w} \\
a_{1}+a_{2}=1
\end{array}\right.
$$

And $k_{w}$ is peak data of wind power output deviation. The article consider that wind power predictive error $e_{w p, t}$ and the reserve requirement of wind power output error are drawn from the same distribution.

$$
f_{t}^{\text {reserve }}\left(e_{w p}\right)=f_{t}\left(e_{w p}\right)
$$

where, $f_{t}^{\text {reserve }}\left(e_{w p}\right)$ is density function of reserve requirement and $f_{t}\left(e_{w p}\right)$ is density function of predictive. And reserve capacity can be determined by preassigned credibility $\alpha$.

$$
\int_{R_{t}^{-}}^{R_{t}^{+}} f_{t}\left(e_{w p}\right) \mathrm{d} \xi>\alpha
$$

where, $\alpha$ is the lower confidence bound; $R_{t}^{+}$and $R_{t}^{-}$represent upper and lower bound of reserve capacity.

\section{DED for Wind Power System}

During researching the DED of wind power system, making wind power scheduling should be the first step which means that we should consider the wind farm power output first. 


\subsection{Objective Function}

$$
C_{G}\left(P_{i}(t)\right)=\sum_{i=1}^{N} U_{i}(t) F_{i}(t)
$$

where, $F_{i}(t)$ is the generation cost of generator $i$ at hour $t ; U_{i}(t)$ is operating state of generator $i$ at hour $t ; 1$ represent running state and 0 represent stopping state; $T$ is the total time for scheduling cycle; $N$ is number of generators.

$$
F_{i}(t)=\left(a_{i}+b_{i} P_{i}(t)+c_{i} P_{i}(t)^{2}\right)
$$

where, $a_{i}, b_{i}, c_{i}$ are coefficients of cost function; $P_{i}(t)$ is active power output of generator $i$ at hour $t$.

\subsection{Constraint}

\subsubsection{Constraints of Wind Farm}

$$
\begin{aligned}
& P_{W \max }=N_{W} K_{W} P_{W}(t) \\
& 0 \leq P_{W}(t) \leq P_{W \max } \\
& P_{W}(t-1)-P_{W}(t) \leq P_{W \mathrm{dn}} \\
& P_{W}(t)-P_{W}(t-1) \leq P_{\text {Wup }} \\
& P_{W \mathrm{dn}}=\min \left\{\gamma \% P_{W R}, \sum_{i=1}^{N}\left[P_{i \max }(t)-P_{i}(t-1)\right]\right\} \\
& P_{\text {Wup }}=\min \left\{\beta \% P_{W R}, \sum_{i=1}^{N}\left[P_{i}(t-1)-P_{i \min }(t)\right]\right\}
\end{aligned}
$$

where, $P_{W \max }$ represent the maximum available output of wind farm at hour $t ; P_{w}(t)$ is maximum available output of wind turbine at hour $t ; K_{w}$ is effective coefficient; $N_{w}$ is number of wind turbines for wind farm. $P_{i \max }(t)$ and $P_{i \min }(t)$ represent the maximum and minimum output of generator $i$ at hour $t . \quad \gamma \%$ and $\beta \%$ are the limitation of change rates.

\subsubsection{Constraints of Conventional Unit}

$$
\begin{aligned}
& P_{G i \min }(t) U_{i}(t) \leq P_{G i}(t) \leq P_{G i \max }(t) U_{i}(t) \\
& P_{i \min }(t)=\max \left\{P_{G i \min }, P_{G i}(t-1)-r_{\text {idown }} \cdot T_{60}\right\} \\
& P_{i \max }(t) \min \left\{P_{G i \max }, P_{G i}(t-1)+r_{\text {iup }} \cdot T_{60}\right\} \\
& \left(X_{i}^{\text {on }}(t)-T_{i}^{\text {on }}\right)\left(U_{i}(t-1)-U_{i}(t)\right) \geq 0 \\
& \left(X_{i}^{\text {off }}(t)-T_{i}^{\text {off }}\right)\left(U_{i}(t)-U_{i}(t-1)\right) \geq 0 \\
& U_{i}(t) U_{i}(t-1)\left[P_{i}(t)-P_{i}(t-1)\right] \leq P_{\text {iup }} \\
& U_{i}(t) U_{i}(t-1)\left[P_{i}(t-1)-P_{i}(t)\right] \leq P_{\text {idown }}
\end{aligned}
$$

where, $P_{i \max }$ and $P_{i \min }$ represent the output limitation of generator $i$; $P_{i \text { down }}$ and $P_{i \text { iup }}$ represent the ramp rate limitation; $X_{\text {ion }}(t)$ and $X_{\text {ioff }}(t)$ represent the cumulative time of running and outage for generator $i$ at hour $t . T_{\text {ion }}$ and $T_{\text {ioff }}$ represent the minimum bound of running and outage cumulative time. 


\subsubsection{Constraints of System Security}

$$
\begin{aligned}
& P_{L}(t)=\sum_{j=1}^{N} P_{G i}(t)+\sum_{j=1}^{D} P_{W j}(t)+P_{\text {loss }} \\
& P_{G m}-P_{L m}-V_{m} \sum_{k=1}^{n b} V_{k}\left(G_{m k} \cos \theta_{m k}+B_{m k} \sin \theta_{m k}\right)=0 \\
& Q_{G m}-Q_{L m}-V_{m} \sum_{k=1}^{n b} V_{k}\left(G_{m k} \sin \theta_{m k}-B_{m k} \cos \theta_{m k}\right)=0 \\
& \theta_{m \text { min }} \leq \theta_{m} \leq \theta_{m \text { max }} \\
& V_{m \text { min }} \leq V_{m} \leq V_{m \text { max }} \\
& S_{m k}^{2} \leq S_{m k \max }^{2}
\end{aligned}
$$

where, $P_{L}(t)$ is total load at hour t; $P_{\text {loss }}$ is power net loss; $n b$ is the number of branches; $m$ and $k$ are heading node number of branch; $V_{k}$ and $\theta_{k}$ are node voltage; $S_{m k}$ is branch power; $S_{m k \max }$ is branch power limitation.

\subsubsection{Constraints of Wind Spinning Reserve}

Constraints of wind power spinning reserve consist of wind farm output optimal method and reserve capacity calculation which can be described by Equations (1)-(6).

\section{Solution Method}

Differential Evolution algorithm is a randomized parallel search algorithm while interior-point method is suitable for solving convex optimization problem. The paper apply interior-point nested differential evolution method to solve the dynamic economic dispatch problem. The steps are as follow.

Step 1. Set related coefficients of differential evolution algorithm;

Step 2. Initial group, each individual can be regard as a solution to the DED problem which consist with power output of thermal generators in the scheduling cycle; and initial group can calculate as follow.

$$
P_{i t}=P_{i}^{\min }+r_{s} \cdot\left(P_{i}^{\max }-P_{i}^{\min }\right)
$$

where, $r_{s}$ is a $[0,1]$ uniform distribution random number.

Step 3. Using interior-point method to solve the OPF for each single individual.

$$
G F=\min \sum_{i=1}^{N}\left[F\left(P_{i}(t)\right)+h_{t} \cdot E\left(P_{i}(t)\right)\right]
$$

Step 4. Evaluate the fitness value of each individual.

$$
T C=\sum_{t=1}^{T} G F
$$

Step 5. Set the iteration number $k+1$;

Step 6. Variation, generate variate individual.

$$
v_{i}^{G}=P_{k}^{G}+F\left(P_{l}^{G}-P_{m}^{G}\right)
$$

where, $P_{k}^{n}, P_{l}^{n}$ and $P_{m}^{n}$ are random selected individuals different from each other; $F$ is zoom factor;

Step 7. Crossover, generate temp individual from original group and variate group $u_{i}^{n}$.

$$
u_{i}^{n}= \begin{cases}v_{i, j}^{n} & \operatorname{rand}(j) \leq C_{R} \text { or } j=\operatorname{rand} n(i) ; \\ P_{i, j}^{n} & \operatorname{rand}(j)>C_{R} \text { and } j \neq \operatorname{rand} n(i) .\end{cases}
$$

where, $\operatorname{rand}(j)$ is random number between [0,1]; and $C_{R}$ is corssover probability;

Step 8. Using interior-point method to solve OPF with $u_{i}^{n}$ to be the initial value, and evaluate the fitness. 
Step 9. Select the next generation according to the greedy method.

$$
P_{i}^{n+1}= \begin{cases}u_{i}^{n} & T C\left(u_{i}^{n}\right)<T C\left(P_{i}^{n}\right) \\ P_{i}^{n} & T C\left(u_{i}^{n}\right) \geq T C\left(P_{i}^{n}\right)\end{cases}
$$

Step 10. Loop over all these individuals and $k=k+1$. If $k$ is less than iteration limitation, turn to step5;

Step 11. Output the optimal solution in group.

\section{Numerical Examples}

Text on the IEEE-RTS24 buses system with two wind farms of 200 MW connected in node 8 and 17. Parameters of generators and load are shown in [10] [11]. Wind speed data adopts from the Royal Netherlands Meteorological Institute (KNMI) history data in 24 hours [12]. Spinning reserve cost coefficient is set to 20\$/MWh [13]. Assume that base load is allocated to 22 and 23 nodes in the context of priority scheduling wind power. As for differential evolution algorithm, population size set to 20 , maximum evolution is 10 , zoom factor is 0.75 and crossover probability factor is set to 1 .

The paper analyses the influence of spinning reserve constraint to dynamic economic dispatch by separate the problem to six situations.

a. No wind power connected;

b. Ignoring spinning reserve constraint with wind power connected;

c. Considering system spinning reserve constraint with wind power connected;

d. Ignoring spinning reserve constraint with limited wind power connected

e. Considering system spinning reserve constraint with limited wind power connected;

f. Considering spinning reserve constraint of wind farms and system with limited wind power connected;

Calculation results of single time continuous optimization with OPF nested DE applied are shown as follow (Tables 1 and 2).

\section{Conclusions}

The paper proposed the DED model considering constraints of security and spinning reserve. Differential evolution algorithm and interior-point method are employed to analyses the influence of wind farm spinning reserve to power system operating scheduling.

1) After considering security and system spinning reserve constraints, the economic dispatch cost increases which better conforms to the wind power system actual operating rule.

Table 1. Result of OPF nested DE method.

\begin{tabular}{ccccc}
\hline \multicolumn{2}{c}{ Solution } & Fuel Cost $\left(10^{6} \$\right)$ & Reserve Cost $\left(10^{6} \$\right)$ & Total Cost $\left(10^{6} \$\right)$ \\
\hline a & 0 & 2.1372 & 0 & 2.1372 \\
& 1 & 2.2075 & 0 & 2.2075 \\
$\mathrm{~b}$ & 0 & 1.8487 & 0 & 1.8487 \\
& 1 & 1.9358 & 0 & 1.9358 \\
$\mathrm{C}$ & 0 & 1.8446 & 0.1306 & 1.9752 \\
$\mathrm{~d}$ & 1 & 1.9193 & 0.1086 & 2.0279 \\
& 0 & 1.8768 & 0 & 1.8768 \\
$\mathrm{e}$ & 1 & 1.9490 & 0 & 1.9490 \\
$\mathrm{f}$ & 0 & 1.8710 & 0.1234 & 1.9944 \\
& 1 & 1.9460 & 0.1054 & 2.0514 \\
\hline
\end{tabular}


Table 2. Result of single time continuous optimal.

\begin{tabular}{ccccc}
\hline \multicolumn{2}{c}{ Solution } & Fuel Cost $\left(10^{6} \$\right)$ & Reserve Cost $\left(10^{6} \$\right)$ & Total cost $\left(10^{6} \$\right)$ \\
\hline a & 0 & 2.1372 & 0 & 2.1372 \\
b & 1 & 2.2378 & 0 & 2.2378 \\
& 0 & 1.8487 & 0 & 1.8487 \\
c & 1 & 1.9410 & 0 & 1.9410 \\
& 0 & 1.8446 & 0.1306 & 1.9752 \\
$\mathrm{~d}$ & 1 & 1.9227 & 0.1038 & 2.0265 \\
& 0 & 1.8768 & 0 & 1.8768 \\
$\mathrm{e}$ & 1 & 1.9640 & 0 & 1.9640 \\
$\mathrm{f}$ & 1 & 1.8710 & 0.1234 & 1.9944 \\
& 0 & 1.9547 & 0.0973 & 2.0520 \\
\hline
\end{tabular}

2) Compared to the largest output mode, it will decrease the system spinning reserve cost to limit the output of wind farms and employ the wind farm spinning reserve.

3) It will reduce the abandon volume of wind, save system reserve capacity and increase the efficiency of the system operating while considering wind farm spinning reserve.

\section{References}

[1] Lei, Y., Wang, W., Hua, Y., et al. (2002) Wind Power Value Analysis on Power System. Power System Technology, 26, 10-14.

[2] Wang, J., Wang, X. and Wu, Y. (2005) Operating Reserve Model in Power Market. IEEE Transactions on Power System, 20, 223-229. http://dx.doi.org/10.1109/TPWRS.2004.841232

[3] Wang, D., Chen, Z., Tu, M., et al. (2012) Reserve Capacity Calculation Considering Large Scale Wind Power. Automation of Electric Power System, 36, 24-28.

[4] Jin, H., Xiong, X. and Wu, Y. (2012) Short-Term Load Forecasting Method Based on Similarity Principle. Automation of Electric Power System, 25, 45-48.

[5] Lee, T. (2007) Optimal Spinning Reserve for Wind Thermal Power System Using EIPSO. IEEE Transactions on Power System, 22, 1612-1621. http://dx.doi.org/10.1109/TPWRS.2007.907519

[6] Hans, B. and Jose, A. (2008) Statistical Analysis of Wind Power Forecast Error. IEEE Transactions on Power System, 23, 983-991. http://dx.doi.org/10.1109/TPWRS.2008.922526

[7] Yao, Y. and Yu, J. (2011) Wind Power System Multi-Objective Hybrid Optimal Scheduling. Automation of Electric Power System, 35, 118-124.

[8] Ortega, M. and Kirscher, D. (2009) Estimating the Spinning Reserve Requirements in Systems with Significant Wind Power Generation Penetration. IEEE Transactions on Power System, 24, 114-124. http://dx.doi.org/10.1109/TPWRS.2008.2004745

[9] Sun, C., Zhou, H. and Zhang, Y. (2012) Dynamic Environment Economic Based on Differential Evolution Algorithm. Computing Science, 11, 208-211.

[10] Basu, M. (2011) Economic Environmental Dispatch Using Multi-Objective Differential Evolution. Applied Soft Computing, 11, 2845-2853. http://dx.doi.org/10.1016/j.asoc.2010.11.014

[11] Basu, M. (2008) Dynamic Economic Emission Dispatch Using Non-Dominated Sorting Genetic Algorithm-II. Electrical Power and Energy Systems, 30, 140-149. http://dx.doi.org/10.1016/j.ijepes.2007.06.009

[12] KNMI Hydra Project (2013) Wind Climate Assessment of the Netherlands. http://dcr.rpi.edu/commdesign/class1.html

[13] Wei, Z., Sun, H., Gu, H., et al. (2012) Wind Power System Dynamic Economic Dispatch Considering Risk Reserve Constraints. Proceedings of the CSEE, 32, 47-55. 